\title{
New Theoretical Results about the Mass Spectrum of Elementary Particles
}

\author{
L.G. Sapogin \\ Department of Physics, Technical University (MADI) Leningradsky \\ pr. 64, A-319, 125829, Moscow, Russia \\ Tel: 7-499-155-04-92 E-mail: sapogin@cnf.madi.ru \\ Yu.A. Ryabov \\ Department of Mathematics, Technical University (MADI) Leningradsky \\ pr. 64, A-319, 125829, Moscow, Russia
}

Tel: 7-499-155-04-36 E-mail: ryabov@vmat.madi.ru

\begin{abstract}
The particle is represented by the wave packet in nonlinear space-time continuum. Because of dispersion, the packet periodically appears and disappears in movement and the envelope of the process coincides with the wave function. There was considered the partial differential equation of telegraph-type describing the motion of such wave packet in spherical coordinate space $(r, \theta, \varphi)$. There was constructed also the analytical solution $u(r, \theta, \varphi)$ of this equation and the integral over all space of $\left.\left.|\operatorname{grad}| u\right|^{2}\right|^{2}$ was supposed being equal to the mass of the particle identified with the wave packet. As the solution $u(r, \theta, \varphi)$ depends on two parameter $L, m$ being positive integer, it was possible to calculate our theoretical particle masses $M_{L m}$ for different $L, m$. So, we have obtained the theoretical mass spectrum of elementary particles. The comparison with known experimental mass spectrum shows our calculated theoretical mass spectrum is sufficiently verisimilar.
\end{abstract}

Keywords: Unitary, Quantum, Wave packet, Mass spectrum, Elementary particles

\section{Introduction}

In the standard quantum theory, a micro-particle is described with the help of a wave function with a probabilistic interpretation. This does not follow from the strict mathematical formalism of the nonrelativistic quantum theory, but is simply postulated. A particle is represented as a point that is the source of a field, but can not be reduced to the field itself and nothing can be said about its "structure" except with these vague words. Modern quantum field theory can not even formulate the problem of finding a mass spectrum.

This dualism is absolutely not satisfactory as the two substances have been introduced, that is, both the points and the fields. Presence of both points and fields at the same time is not satisfactory from general philosophical positions - "razors of Ockama". Besides that, the presence of the points leads to non-convergences, which are eliminated by various methods, including the introduction of a re-normalization group that is declined by many mathematicians and physicists, for example, P.A.M. Dirac.

The original idea of Schroedinger was to represent a particle as a wave packet of de Broglie waves. As he wrote in one of his letters, he "was happy for three months" before British mathematician Darwin showed that such packet quickly and steadily dissipates and disappears. So, it turned out that this beautiful and unique idea to represent a particle as a portion of a field is not realizable in the context of wave packets of de Broglie waves. Later, de Broglie tried to save this idea by introducing nonlinearity for the rest of his life, but wasn't able to obtain significant results. It was proved [Lyamov V.E., 1969] by V.E. Lyamov and L.G. Sapogin in 1968 that 
every wave packet constructed from de Broglie waves with the spectrum a(k) satisfying the condition of Viner-Pely (the condition for the existence of localized wave packets)

$$
\int_{-\infty}^{\infty} \frac{|\ln (\mathrm{a}(\mathrm{k}))|}{1+\mathrm{k}^{2}} \geq 0
$$

becomes blurred in every case.

There is a school in physics, going back to William Clifford, Albert Einstein, Erwin Schrödinger and Louis de Broglie, where a particle is represented as a cluster or packet of waves in a certain unified field. According to M. Jemer's classification, this is a 'unitary' approach. The essence of this paradigm is clearly expressed by Albert Einstein's own words: "We could regard substance as those areas of space where a field is immense. From this point of view, a thrown stone is an area of immense field intensity moving at the stone's speed. In such new physics there would be no place for substance and field, since field would be the only reality... and the laws of movement would automatically ensue from the laws of field.»

However, its realization appeared to be possible only in the context of the Unitary Quantum Theory (UQT) within last two decades. It is impressive, that the problem of mass spectrum has been reduced to exact analytical solution of a nonlinear integro-differential equation. In UQT the quantization of particles on masses appears as a subtle consequence of a balance between dispersion and nonlinearity, and the particle represents something like a very little water-ball, the contour of which is the density of energy.

Following, in essence, this general idea, the Unitary Quantum Theory (UQT) represents a particle as a bunched field (cluster) or a packet of partial waves with linear dispersion, and the particle is identified with some field. Dispersion is chosen in such a way that the wave packet would periodically disappear and appear in movement, and the envelope of the process would coincide with de Broglie wave. Based on this idea, the relativistic-invariant model of such unitary quantum field theory was built.

The relativistic invariant equation for our wave packet is following [Boichenko V.A., 1984; Sapogin L.G., 2003; 2005; 2008]:

$$
i \lambda^{\mu} \frac{\partial \Phi}{\partial x^{\mu}}-\frac{c \Phi}{\hbar} \int\left(\bar{\Phi} \lambda_{1} u^{\mu} \frac{\partial \Phi}{\partial x^{\mu}}-u^{\mu} \frac{\partial \bar{\Phi}}{\partial x^{\mu}} \lambda_{1} \Phi\right) \frac{d V}{\gamma}=0
$$

where $\Phi$ is the function of coordinates $x^{\mu}=(c t, x), \mu=0,1,2,3$, describing different characteristics of our wave packet, $u^{\mu}=\left(\frac{1}{\gamma}, \frac{v}{\gamma}\right)$ is the four-velocity of the particle, $\lambda_{1}$ is some number matrix and matrices $\lambda^{\mu}(32 \times 32)$ satisfy the commutation relations

$$
\lambda^{\mu} \lambda^{v}+\lambda^{v} \lambda^{\mu}=2 g^{\mu v} I, \mu, v=0,1,2,3
$$

where $g^{\mu v}$ is the metrical tensor. This fundamental equation of UQT describes, in our opinion, all properties of elementary particles. It is possible to derive from (1) the Dirac equation and also the relativistic invariant Hamilton - Jacoby equation [Sapogin L.G., 1988; 1991; 2003; 2005; 2008]. We have succeeded in solving only the simplified scalar variant of eq. (1). However, the solution obtained has allowed to determine theoretically [Sapogin L.G., 1988; 1991; 2003; 2005; 2008] the elementary electrical charge and the fine-structure constant $\alpha$ with high precision (our theoretical value $\alpha=1 / 137.962$ ), the known experimental value $\alpha=1 / 137.03552$ Our efforts to find more complete solution of eq.(1) were unsuccessful. Note, our approach based on Unitary Quantum Theory has nothing in common with Standard Model of Elementary Particles.

\section{Common approaches}

Nevertheless, our idea to consider a particle as some moving wave packet which periodically disappears and appears in movement, has allowed to arrive to the conclusion [Sapogin L.G., 2005; 2008] that such particle may be described by the common telegraph - type equation of second order. In one-dimension case this equation is following: 


$$
\frac{1}{v^{2}} \frac{\partial^{2} F(x, t)}{\partial t^{2}}-\frac{\partial^{2} F(x, t)}{\partial x^{2}}-\frac{2 i m c^{2} \sqrt{1-\frac{v^{2}}{c^{2}}}}{\hbar v^{2}} \frac{\partial F(x, t)}{\partial t}-\frac{m^{2} c^{4}}{\hbar^{2} v^{2}}\left(1-\frac{v^{2}}{c^{2}}\right) F(x, t)=0
$$

Note, this equation would be relativistic invariant if the root $\sqrt{1-v^{2} / c^{2}}$ would be placed in denominator.

Equation (2) is satisfied exactly by relativistic invariant solutions in the form of a standard planar quantum-mechanical wave and also in the form of disappearing and appearing wave-packet, viz.,

$$
F(x, t)=\exp \left(\frac{i}{\hbar} \frac{m c^{2} t-m v x}{\sqrt{1-\frac{v^{2}}{c^{2}}}}\right)
$$

or

$$
F(x, t)=\exp \left(\frac{i}{\hbar} \frac{m c^{2} t-m v x}{\sqrt{1-\frac{v^{2}}{c^{2}}}}\right) \varphi(x-v t),
$$

where $\varphi$ is an arbitrary function of its argument $x-v t$.

We will show that eq. (2) (considered in the case of 3-dimension coordinate $\operatorname{space}(r, \theta, \varphi))$ allows, namely, to determine theoretically the mass spectrum of elementary particles.

Such equation for the function $u=u(r, \theta, \varphi)$ is following:

$$
\begin{gathered}
\frac{1}{v^{2}} \frac{\partial^{2} u}{\partial t^{2}}-\frac{1}{r^{2} \sin \theta}\left(2 r \sin \theta \frac{\partial u}{\partial r}+r^{2} \sin \theta \frac{\partial^{2} u}{\partial r^{2}}+\cos \theta \frac{\partial u}{\partial \theta}+\sin \theta \frac{\partial^{2} u}{\partial \theta^{2}}+\frac{1}{\sin \theta} \frac{\partial^{2} u}{\partial \varphi^{2}}\right)-\frac{2 i M c^{2} \sqrt{1-\frac{v^{2}}{c^{2}}} \frac{\partial u}{\partial t}-}{v^{2} \hbar} \\
-\frac{M^{2} c^{4}}{v^{2} \hbar^{2}}\left(1-\frac{v^{2}}{c^{2}}\right) u=0,
\end{gathered}
$$

(the symbol $m$ is replaced by $M$ ).

We will use the natural system of units and put $\hbar=1, c=1$, and will seek the solution of eq. (5) in the following form:

$$
u=\frac{f}{r} \exp \left(\frac{i M t}{\sqrt{1-v^{2}}}-\frac{i M v r}{\sqrt{1-v^{2}}}\right)
$$

where $f=f(r, \theta, \varphi)$ is some function not depending on $t$. This function represents as hardened wave packet in coordinate space $(r, \theta, \varphi)$. Substituting (6) in eq. (5), we get

$$
\begin{aligned}
2 i M v r^{2} \cos ^{2} \theta \frac{\partial f}{\partial r}-2 i M v r^{2} & \frac{\partial f}{\partial r}+r^{2} \sqrt{1-v^{2}} \frac{\partial^{2} f}{\partial r^{2}} \sin ^{2} \theta+\sqrt{1-v^{2}} \frac{\partial^{2} f}{\partial \theta^{2}} \sin ^{2} \theta+ \\
& +\sqrt{1-v^{2}}\left(\frac{\partial^{2} f}{\partial \varphi^{2}}+\sin \theta \cos \theta \frac{\partial f}{\partial \theta}\right)=0 .
\end{aligned}
$$

We will seek the solution of eq. (7) in form:

$$
f=R(r) Y_{L m}(\theta, \varphi),
$$

where 


$$
Y_{L m}(\theta, \varphi)=\frac{\sqrt{(2 L+1)(L-m) !}}{2 \sqrt{\pi(L+m) !}} P_{L}^{m}(\cos \theta) \exp ( \pm i m \varphi)
$$

$P_{L}^{m}(\cos \theta)$ is the Legendre function, $Y_{L m}(\theta, \varphi)$ is the Spherical Harmonic and $L, m$ are nonnegative integers $L=0,1,2,3, \ldots, \quad m=0 \pm 1 \pm 2 \pm 3$. . besides $m \leq L$. Substituting (8) in eq. (7), we come to the following equation with respect to the function $R(r)$ :

$$
\left(\frac{d^{2} R(r)}{d r^{2}} r^{2} \sqrt{1-v^{2}}-2 i \frac{d R(r)}{d r} M v r^{2}\right)-R(r) L^{2} \sqrt{1-v^{2}}-R(r) L \sqrt{1-v^{2}}=0
$$

The solution $R(r)=R_{L}(r)$ of this equation depends on parameter $L$ and we obtain the family of solutions $u_{L m}(r, \theta, \varphi, t)$ of equation (5) depending on parameters $L, m$ and describing corresponding partial wave-packets.

It is natural to suppose that the modulus of every solution $u_{L m}$ describes the amplitude of the world unitary potential $\Phi_{L m}$ determined by this equation, and the world potential itself is represented by the quadrate of amplitude modulus, i.e.

$$
\Phi_{L m}=\left|u_{L m}\right|^{2}=\left|\frac{R_{L}(r)}{r} Y_{L m}(\theta, \varphi)\right|^{2}
$$

Further, we consider the gradient of this potential as the tension of corresponding field (it is the custom in electrodynamics) of the partial wave packet and consider the quadrate of the tension as the density $W_{L m}$ of the energy or of the wave packet's mass distributed continuously in space. So, the mass $M=M_{L m}$ of our partial wave packet may be determined as the integral of density $W_{L m}$ over all space $(r, \theta, \varphi)$ :

$$
M=\int_{0}^{\infty} \int_{0}^{\pi} \int_{0}^{2 \pi} W_{L m} r^{2} \sin \theta d r d \theta d \varphi
$$

where $W_{L m}=\left|\operatorname{grad} \Phi_{L m}\right|^{2}$. We rewrite the equation (9) in form:

$$
2 i v M=\frac{1}{r^{2} R^{\prime}(r)}\left(R^{\prime \prime}(r) r^{2}-L(L+1) R(r)\right) \sqrt{1-v^{2}}, \quad\left('=\frac{d}{d r}\right) .
$$

We consider the mass of the wave packet as its inner (proper) characteristic not depending on the velocity of its movement. Now, replacing the mass by the integral, and next differentiating both parts of equality and supposing $v=0$ obtain the following differential equation for $R(r)$ :

$$
R^{\prime \prime}-\frac{L(L+1)}{r^{2}} R=C R^{\prime},
$$

where $C$ is some constant. This equation possesses the analytical general solution:

$$
R\left(r, C_{1}, C_{2}\right)=C_{1} \exp \left(\frac{C}{2} r\right) \sqrt{r} \mathrm{~J}\left(L+\frac{1}{2}, \frac{1}{2} \sqrt{-C^{2}} r\right)+C_{2} \exp \left(\frac{C}{2} r\right) \sqrt{r} \mathrm{Y}\left(L+\frac{1}{2}, \frac{1}{2} \sqrt{-C^{2}} r\right),
$$

where $C_{1}, C_{2}$ arbitrary constants and $\mathrm{J}$ and $\mathrm{Y}$ are are the Bessel functions. Since we seek the finite solution $R(r)$ for $r \rightarrow 0, r \rightarrow \infty$ and tending to zero for, $r \rightarrow \infty$ we set $C_{2}=0$ and can set some positive value for 
$C_{1}$ and some negative value for the constant $C$ in eq. (13). The calculations show the choice of these constants has influence only on the absolute value of the masses calculated below but the ratios of these masses remain the same. We have chosen the simplest values

$$
C_{1}=1, C=-2
$$

and have obtained following solution

$$
R(r)=\sqrt{r} \exp (-r) \mathrm{J}\left(L+\frac{1}{2}, i r\right)
$$

where $\mathrm{J}\left(L+\frac{1}{2}, i r\right)$ is the Bessel function of $1^{\text {st }}$ type with imaginary argument, or

$$
R(r)=i^{L+\frac{1}{2}} \sqrt{r} \exp (-r) \mathrm{I}\left(L+\frac{1}{2}, r\right),
$$

where $\mathrm{I}\left(L+\frac{1}{2}, r\right)$ is the modified Bessel function of $1^{\text {st }}$ type.

So, we obtain the following expression for the world unitary potential $\Phi_{L m}$ (taking into consideration $(6,8,8$, 10) :

$$
\Phi_{L m}=\frac{e^{-2 r}}{4 \pi r}\left|\frac{(2 L+1)(L-m) ! \mathrm{I}\left(L+\frac{1}{2}, r\right)^{2} \mathrm{P}_{L}^{m}(\cos \theta)^{2}}{(L+m) !}\right|
$$

Now, we form $\operatorname{grad} \Phi_{L m}$ considered as the tension of the field and form also the quadrate of its modulus considered as the mass density $W_{L m}$. We obtain:

$$
\begin{gathered}
W_{L m}=2 e^{-4 r}\left(\frac{(L-m) !^{2} \mathrm{I}\left(L+\frac{1}{2}, r\right)^{2}\left((L+r+1) \mathrm{I}\left(L+\frac{1}{2}, r\right)-r \mathrm{I}\left(L-\frac{1}{2}, r\right)\right)^{2} \mathrm{P}_{\mathrm{L}}^{\mathrm{m}}(\cos \theta)^{4}\left(L+\frac{1}{2}\right)^{2}}{\pi^{2} r^{4}(L+m) !^{2}}+\right. \\
\left.+\frac{\left(L+\frac{1}{2}\right)^{2} \mathrm{I}\left(L+\frac{1}{2}, r\right)^{4}(L-m) !^{2} \mathrm{P}_{\mathrm{L}}^{\mathrm{m}}(\cos \theta)^{2}\left((m-L-1) \mathrm{P}_{L+1}^{\mathrm{m}}(\cos \theta)+(L+1) \cos \theta \mathrm{P}_{\mathrm{L}}^{\mathrm{m}}(\cos \theta)\right)^{2}}{\pi^{2} r^{4}(L+m) !^{2} \sin ^{2} \theta}\right)
\end{gathered}
$$

The integrals of $W_{L m}$ over all spherical space $(r, \theta, \varphi)$ for different $L=0,1,2, \ldots$ and $m=0, \pm 1, \pm 2, \ldots, m \leq L$ is equal to required different masses $M_{L m}$ of elementary particles, i.e.

$$
M_{L m}=\int_{0}^{\infty} \int_{0}^{\pi} \int_{0}^{2 \pi} W_{L m} r^{2} \sin (\theta) d r d \theta d \varphi
$$

Since $W_{L m}$ do not depend on $\varphi$ and the Legendre functions in expressions of $W_{L m}$ may be integrated analytically, we calculated, at first, analytically (with help of Mathematica-7) the integrals

$$
U_{L m}=\int_{0}^{\pi} W r^{2} \sin (\theta) d \theta \int_{0}^{2 \pi} d \varphi=2 \pi \int_{0}^{\pi} W r^{2} \sin (\theta) d \theta
$$

and then calculated numerically (with the help of Mathematica-7) the integrals

$$
M_{L m}=\int_{0}^{\infty} U_{L m} d r
$$

\section{Results}

For example, we have obtained for $L=0$ и $m=0$ : 


$$
U_{0,0}=\frac{8 e^{-4 r} \sinh (r)^{2}}{\pi^{3} r^{4}}\left\{\left(r^{2}+\frac{1}{2}+r\right) \cosh (r)^{2}-r(1+r) \sinh (r) \cosh (r)-\frac{(1+r)^{2}}{2}\right\}
$$

and

$$
M_{0,0}=\int_{0}^{\infty} U_{0,0} d r=0.00394436469
$$

For $L=1, m=1$

$$
\begin{aligned}
& U_{11}= \frac{8 e^{-4 r}}{\pi^{3} r^{8}}\left[\left(r^{6}+5 r^{5}+\frac{93}{8} r^{4}+13 r^{3}+\frac{61}{4} r^{2}+2 r+\frac{17}{8}\right) \cosh ^{4} r-\right. \\
&-r \sinh r \cosh ^{3}\left(r^{5}+5 r^{4}+11 r^{3}+\frac{33}{2} r^{2}+8 r+\frac{17}{2}\right)- \\
&- \cosh ^{2} r\left(\frac{1}{2} r^{6}+3 r^{5}+10 r^{4}+14 r^{3}+\frac{71}{4} r^{2}+4 r+\frac{17}{4}\right)+ \\
&\left.+r \sinh r \cosh r\left(r^{4}+3 r^{3}+8 r^{2}+8 r+\frac{17}{2}\right)+\frac{1}{2} r^{4}+r^{3}+\frac{5}{2} r^{2}+2 r+\frac{17}{8}\right]
\end{aligned}
$$

and

$$
M_{1,1}=0.00006798678730
$$

The calculations for small values of $L$ are sufficiently simple. But for large $L$, the quantities $U_{L m}$ are represented by long polynomials in $r$ and $\cosh (r), \sinh (r)$ with enormous numerical coefficients and the integration of these polynomials meets serious technical difficulties.

We consider the ensemble $L+1$ particles (masses) with given $L$ and $m=0 \ldots \pm L$ to be one family and we will use the notations $M_{L, 0}, M_{L, 1}, \ldots, M_{L, L}$ for particles (masses) of the family with given $L$. We have calculated and analyzed in full the masses of 49 families $(L=0, \ldots, 48)$, i.e. of 1225 particles. Our PC with $3 G H z, R A M=4 G B$ has required for these calculations nearly 1 weeks of computing time.

We have compared our theoretical spectrum for 1225 masses with known experimental spectrum for elementary particles measured in $M e V$. The zero-point for the matching of both spectra was required. We have taken for such matching the quotient of the muon mass to the electron mass. As we know, this quotient for observed muons and electrons is measured experimentally [W. Liu, 1999] with the most precision and is equal 206.768283(10). Each our calculated mass was divided consecutively by all other 1224 masses and the resulting quotients were compared with the mentioned number. It turned out that the quotient of our masses $M_{16,10} / M_{48,45}$ is equal to 206.7607796 (with relative divergence $0.0039 \%$ ) and we have taken our mass $M_{48,45}$ equal to $0.2894982442536304 \cdot 10^{-10}$ for zero-point, i.e. for our electron mass. After, there were divided all other 1224 masses $M_{L, m}$ by $M_{48,45}$ and we have obtained our theoretical spectrum in electron masses which may be compared (after expressing in $\mathrm{MeV}$ ) with known experimental masses. Here is the table 1 with our masses $M_{L m}$ for 33 cases of the well coincidence with well known experimental values (relative errors are less than $1 \%$ in 30 cases and between $1.3 \%$ and $1.8 \%$ in three cases). 
Note, the ratio of our proton mass $M_{12,1}$ and our electron mass $M_{48,45}$ is equal 1832.355 with relative error

$0.207 \%$ in comparison with well known experimental ratio 1836.152167 . Our calculated spectrum containing 169 masses from muon to the heaviest mass approximates also others well known particles and, although the coincidences with experimental data are worse but quite acceptable (with relative divergences not more than several per cent). The mass values for negative $m$ coincides with the mass valued for positive $m$ (antiparticles?).

On the whole, this table shows the striking coincidence of our theoretical values with essential quantity of the known experimental masses and, by no means, such coincidence may be called occasional. The probability of such occasional coincidence is less $10^{-60}$. Note, the choice of the nominee for the electron's mass is not unique and may be further calculations of families with $L=60 \ldots .100$ would allow to obtain the better result. Our calculated theoretical spectrum contains also the values near to the masses of quarks. The experimental data for quarks are not so precise and are determined in an indirect way. We give the separate table 2 with the calculated and experimental quark masses.

We have carried out also the series of calculations $M_{L m}$ for $L$ exceeding 48 including $L=60$. The ratio of maximal $M_{0,0}=0.0039443641689$ to minimal $M_{60,60}=0.3909395521 \cdot 10^{-11}$ is of order $10^{9}$. The ratio of maximal $M_{0,0}$ to the mass $M_{12,1}=0.5304640719 \cdot 10^{-7}$ of proton is equal 74400 . This number does not contradict the known the experimental data.

Note, the radial function $U_{L m}(r)$ being the density mass as function of $r$, is equal zero always for $r=0$ and for all $L, m$, and, at first, increases very swiftly on the right from for $r=0$ and then very swiftly decreases. The plot of $U_{L m}(r)$ reminds for large $L$ quasi delta-function approaching to coordinates origin as $L$ increases (very simplified analogy is shown on Figure 1).

Such theoretical model describes a particle as very small bubble in space-time continuum cut by spherical harmonics. Curious, such model, namely, was considered by A. Poincare [Poincare A., 1974].

Certainly, we do not intend to assert that our results are adequate in full to the known experimental mass spectrum of elementary particles. The divergences are present. Our theoretical spectrum contains the large quantity (1053) of masses between electron mass and muon mass but such real particles are not observed till now.

Our spectrum contains many light particles $M_{L, m}(L>48)$ with masses differing extremely little one from another.

It may be supposed there is exists quasi-continuous distribution of lightest particles not affirmed till now by experiments. We suppose that this region of our calculated spectrum contains also the values corresponding to masses of all 6 neutrinos, and it will be possible to discover their theoretical masses after sufficiently precise experimental determination of their masses.

Our spectrum contains 169 particles from the muon to the heaviest particle $M_{0,0}$ but there is observed the large quantity of particles in this interval with short "life-time" (so called "resonances") of order $10^{-22} \mathrm{sec}$. These divergences require the further researches. With respect to light particles, it may be supposed there are exist some selection principles (not discovered till now theoretically) for such particles and these principles lead to essential decreasing of particles quantity between muons and electrons. We suppose that such principles arise 
theoretically from some relations between the tensors of different valences (ranks) and spherical functions for different $L, m$ and leave this complicate problem for future researches. On the other hand the presence of more than a thousand of light particles between the electron and muon can be explained by the numerous coupled particles $e-e, e-\mu, \mu-\mu$ and light particles which have less mass than the electron can belong to numerous neutrino and their coupled particles. May be all these light particles constitute the dark matter?

It arise the question with respect to the particles with short "life-time": may we take all these particles for elementary? Our Unitary Quantum Theory allows formulating the following criterion. If the way which the particle (which we identify with appearing and disappearing wave packet) passes from the moment of its appearing to the moment of its destruction is much longer than de Broglie wave, then such particle may be called elementary. Have we reason to call "elementary" the particle with life-time of order $10^{-22} \mathrm{sec}$ ?

Let us point to following essential circumstance. Viz., if we will use the Schrödinger equation in spherical coordinates (relativistic-noninvariant) or Klein-Gordon equation (relativistic-invariant) instead our initial equation (5), then we will come to the same theoretical mass spectrum. Really, the mention Schrödinger equation is following:

$$
\frac{\hbar^{2}}{2} \frac{\left(2 r \sin \theta \frac{\partial u}{\partial r}+r^{2} \sin \theta \frac{\partial^{2} u}{\partial r^{2}}+\cos \theta \frac{\partial u}{\partial \theta}+\sin \theta \frac{\partial^{2} u}{\partial \theta^{2}}+\frac{1}{\sin \theta} \frac{\partial^{2} u}{\partial \varphi^{2}}\right)}{M r^{2} \sin \theta}+i \hbar \frac{\partial u}{\partial t}=0,
$$

where $M$ is the particle's mass. We will seek the solution of this equation in form of unitary wave packet $f$ :

$$
u=\frac{f}{r} \exp \left(-i \frac{M v^{2}}{2 \hbar} t+i \frac{M v}{\hbar} r\right)
$$

where $f=f(r, \theta, \varphi)$ is the function of coordinates and does not depend on the time. The function $u$ is considered as the amplitude of the world unitary potential $\Phi$. Substituting (23) in (22), we obtain (after simplification) following equation

$$
\hbar r^{2} \sin ^{2} \theta \frac{\partial^{2} f}{\partial r^{2}}-2 i M v r^{2} \sin ^{2} \theta \frac{\partial f}{\partial r}+\frac{\hbar}{2} \sin 2 \theta \frac{\partial f}{\partial \theta}+\hbar \sin ^{2} \theta \frac{\partial^{2} f}{\partial \theta^{2}}+\hbar \frac{\partial^{2} f}{\partial \varphi^{2}}=0
$$

This equation coincides with our equation (7) if we put $\sqrt{1-v^{2}}$ instead $\hbar$. The further study described above remains without changes.

Let us consider Klein — Gordon equation in spherical coordinates and in natural units system

$(c=1, \hbar=1)$ :

$$
\frac{\left(2 r \sin \theta \frac{\partial u}{\partial r}+r^{2} \sin \theta \frac{\partial^{2} u}{\partial r^{2}}+\cos \theta \frac{\partial u}{\partial \theta}+\sin \theta \frac{\partial^{2} u}{\partial \theta^{2}}+\frac{1}{\sin \theta} \frac{\partial^{2} u}{\partial \varphi^{2}}\right)}{r^{2} \sin \theta}-\frac{\partial^{2} u}{\partial t^{2}}-M^{2} u=0,
$$

where $M$ is the particle's mass. We will seek the solution

$$
u=\frac{f}{r} \exp \left(\frac{i M t}{\sqrt{1-v^{2}}}-\frac{i M v r}{\sqrt{1-v^{2}}}\right),
$$


where $f=f(r, \theta, \varphi)$ is the function of coordinates not depending explicitly on $t$. Substituting (26) in (25), we obtain following equation after simplification:

$$
r^{2} \sin ^{2} \theta \sqrt{1-v^{2}} \frac{\partial^{2} f}{\partial r^{2}}-2 i v r^{2} M \sin ^{2} \theta \frac{\partial f}{\partial r}+\sin ^{2} \theta \sqrt{1-v^{2}} \frac{\partial^{2} f}{\partial \theta^{2}}+\sqrt{1-v^{2}} \frac{\partial^{2} f}{\partial \varphi^{2}}+\frac{\sqrt{1-v^{2}}}{2} \sin 2 \theta \frac{\partial f}{\partial \theta}=0 .
$$

This equation coincides in full with our equation (7) and we will come to the same results.

So, different initial equations (5), (22), (25) (the last is relativistic invariant and the other two are relativistic non-invariant) lead to the same theoretical mass spectrum. Note the following remarkable fact: the standard theory allowed to detect spectra by using always the quantum equations with outer potential and as corollaries to geometric relations between de Broglie wave's length and characteristic dimension of potential function. The quantum equation of our theory do not contain the outer potential and describe a particle in empty free space; the mass quantization arises owing to the delicate balance of dispersion and non-linearity which provides the stability of some wave packets number. It is the first case when spectra are detected by using the quantum equations without outer potential.

Here is the table 3 with all our theoretical masses from the muon to the heaviest $M_{0,0}(\mathrm{MeV})$.

\section{Conclusion}

In view of all said above, we are bold, nevertheless, to say that our results represent the substantial advancement on the way of solution for the extremely complicated theoretical problem of the mass spectrum for elementary particles and to underline that this advancement is owing to our Unitary Quantum Theory. We hope that further analysis with the help of exact equation (1) of our theory will allow to obtain more precise results.

We would like to propose the name "Dzhan - particle" for our heaviest particle $M_{0,0}$ in honour of the general

Air Force RF astronaut V.A. Dzhanibekov. As we know, particles with mass of such order are observed in cosmic rays.

The authors are thankful to astronaut V.A. Dzhanibekov, to Professors V.M. Dubovik (Dubna, JINR), F.A. Gareev (Dubna, JINR), Yu.L. Ratis (Samara State University) and to translator S.V. Romanova for support of our work and fruitful discussions.

\section{References}

Boichenko V.A., Sapogin L.G. (1984). On the Equation of the Unitary Quantum Theory. Annales de la Fondation Louis de Broglie, vol. 9, No.3, p.221.

Lyamov V.E., Sapogin L.G. (1969). About the motion of wave packets in dispersive medium. Journal Specialnaya radioelectronika, 1, p.17-25, Moscow, (Russian).

Poincare A. (1974). Sur la Dynamique de l'electron, Coll.Works, v. 3, pp.433-515, Moscow. Science, (Russian, transl. from French).

Sapogin L.G. (1973). United field and Quantum Mechanics, System Researches (physical Researches) Acad. Science USSR, Vladivostok, 2, pp. 54-84, (Russian).

Sapogin L.G. (1979). On Unitary Quantum Mechanics. Nuovo Cimento, vol. 53A, No 2, p.251.

Sapogin L.G. (1980). A Unitary Quantum Field Theory. Annales de la Fondation Louis de Broglie, vol.5, No 4, p.285-300.

Sapogin L.G. (1982). A Statistical Theory of Measurements in Unitary Quantum Mechanics. Nuovo Cimento, vol.70B, No.1, p.80.

Sapogin L.G. (1982). A Statistical Theory of the Detector in Unitary Quantum Mechanics. Nuovo Cimento, vol.71B, No. 3, p.246.

Sapogin L.G., Boichenko V.A. (1988). On the Solution of One Non-linear Equation. Nuovo Cimento, vol.102B, No 4, p.433.

Sapogin L.G., Boichenko V.A. (1991). On the Charge and Mass of Particles in Unitary Quantum Theory. Nuovo Cimento, vol.104A, No 10, p.1483. 
Sapogin L.G., Ryabov Yu. A. (2008). On the mass spectrum of elementary particles in Unitary Quantum Theory, The Old and New Concepts of Physics, Vol. 5, No.3.

Sapogin L.G., Ryabov Yu. A., Boichenko V. A. (2008). Unitary Quantum Theory and a New Sources of Energy, Ed. Science-Press, Moscow, (Russian, transl. from English).

Sapogin L.G., Ryabov Yu.A, Boichenko V.A. (2005). Unitary Quantum Theory and a New Sources of Energy, Archer Enterprises, Geneva, NY, USA.

Sapogin L.G., Ryabov Yu.A., Utchastkin V.I. (2003). Unitary Quantum Theory and a New Energy Sources . Ed. MADI, Moscow, (Russian).

W. Liu, M. G. Boshier, S. Dhawan, O. van Dyck, P. Egan, X. Fei, M. G. Perdekamp, V. W. Hughes, M. Janousch, K. Jungmann, D. Kawall, F. G. Mariam, C. Pillai, R. Prigl, G. zu Putlitz, I. Reinhard, W. Schwarz, P. A. Thompson, and K. A. Woodle. (1999). Phys. Rev. Lett. v. 82, 711.

Table 1. Calculated and experimental some elementary particles masses $(\mathrm{MeV})$

$$
\text { (e-electron, } \mu \text {-muon, } \pi^{0}-\pi \text {-meson, } p \text { - proton etc.) }
$$

\begin{tabular}{|c|c|c|c|c|}
\hline$M_{L, m}$ & THEORY & EXPERIMENT & NOTATION & ERROR \% \\
\hline$M_{48,45}$ & 0.51099906 & 0.51099906 & e & -- \\
\hline$M_{16,10}$ & 105.6545640 & 105.658387 & $\mu$ & 0.0036 \\
\hline$M_{18,4}$ & 135.8958708 & 134.9739 & $\pi^{0}$ & 0.683 \\
\hline$M_{23,0}$ & 137.2902541 & 139.5675 & $\pi^{+}, \pi^{-}$ & 1.62 \\
\hline$M_{14,1}$ & 541.7587460 & 548.86 & $\eta$ & 1.29 \\
\hline$M_{7,7}$ & 894.0806293 & 891.8 & $K^{*+}, K^{* 0}$ & 0.25 \\
\hline$M_{12,1}$ & 936.3325942 & 938.2723 & $p$ & 0.206 \\
\hline$M_{10,4}$ & 957.1290490 & 957.2 & $\omega$ & 0.0083 \\
\hline$M_{9,5}$ & 1110.473414 & 1115.63 & $\Lambda$ & 0.462 \\
\hline$M_{8,6}$ & 1224.151552 & 1233 & $b_{1}^{0}$ & 0.71 \\
\hline$M_{11,1}$ & 1271.916682 & 1270 & $K^{*}$ & 0.14 \\
\hline$M_{9,4}$ & 1331.705434 & 1321.32 & $\Xi^{-}$ & 0.78 \\
\hline$M_{10,2}$ & 1378,127355 & 1382.8 & $\sum^{0}$ & 0.33 \\
\hline$M_{12,0}$ & 1524.617683 & 1520.1 & $\Lambda_{2}$ & 0.29 \\
\hline$M_{8,5}$ & 1549.444919 & $1540 \pm 5$ & $F_{1}$ & 0.28 \\
\hline
\end{tabular}




\begin{tabular}{|c|c|c|c|c|}
\hline$M_{7,6}$ & 1595.510637 & 1594 & $\omega_{1}$ & 0.094 \\
\hline$M_{9,3}$ & 1601.282953 & 1600 & $\rho^{\prime}$ & 0.08 \\
\hline$M_{6,6}$ & 1718.917400 & 1720 & $N_{0}^{3}$ & 0.06 \\
\hline$M_{10,1}$ & 1774.917815 & 1777 & $\tau$ & 0.1 \\
\hline$M_{8,4}$ & 1906.842877 & 1905 & $\overline{\Delta_{5}^{+}}$ & 0.096 \\
\hline$M_{9,2}$ & 1965.115639 & 1950 & $\Delta_{4}$ & 0.77 \\
\hline$M_{11,0}$ & 2092.497779 & 2100 & $\Lambda_{4}$ & 0.35 \\
\hline$M_{7.5}$ & 2195.695293 & 2190 & $\mathrm{~N}(2190)$ & 0.25 \\
\hline$M_{7,4}$ & 2818.645188 & 2820 & $\eta_{c}$ & 0.048 \\
\hline$M_{10,0}$ & 2954.549810 & 2980 & $\eta$ & 0.85 \\
\hline$M_{6,5}$ & 3082.979571 & 3096 & $J / \psi$ & 0.42 \\
\hline$M_{7,3}$ & 3543.664516 & 3556.3 & $\chi$ & 0.35 \\
\hline$M_{5,5}$ & 3687.679612 & 3686.0 & $\psi^{\prime}$ & 0.04 \\
\hline$M_{7,2}$ & 4496.650298 & 4415 & $\psi^{\prime \prime \prime}$ & 1.84 \\
\hline$M_{6,4}$ & 5642.230394 & 5629.6 & $\Xi_{b}$ & 0.8 \\
\hline$M_{5,3}$ & 9499.927309 & 9460.32 & $\Upsilon^{\prime}$ & 0.41 \\
\hline$M_{6,1}$ & 10075.78271 & 10023.3 & $\Upsilon^{\prime \prime}$ & 0.523 \\
\hline$M_{7,0}$ & 10533.15222 & 10580 & $\Upsilon^{\cdots}$ & 0.442 \\
\hline$M_{0,0}$ & 69622749 & $?$ & Dzhan & $?$ \\
\hline
\end{tabular}


Table 2. Calculated and experimental quark masses $(\mathrm{MeV})$

\begin{tabular}{||c|c|c||}
\hline \hline$M_{L, m}$ & THEORY & EXPERIMENT \\
\hline$M_{38,16}$ & 5.003455873 & $3-7$ \\
\hline$M_{30,25}$ & 2.75072130 & $1.5-3.0$ \\
\hline$M_{20,4}$ & 94.4251568 & $95 \pm 25$ \\
\hline$M_{11,1}$ & 1271.9166 & $1250 \pm 90$ \\
\hline$M_{6,4}$ & 4300.86662 & $4200 \pm 70$ \\
\hline$M_{3,0}$ & 179100 & $178000 \pm 4300$ \\
\hline
\end{tabular}

Table 3. All theoretical masses from the muon to the heaviest $M_{0,0}(\mathrm{MeV})$

105.655, 105.94, 106.241, 108.291, 108.997, 109.597, 110.133, 112.784, 117.054, 118.136, $120.31,121.826,122.664,125.522,125.71,127.187,127.237,127.306,131.445,133.013$, $135.896,137.29,142.287,144.326,145.96,147.309,147.698,149.62,149.905,153.765$, $153.827,159.796,162.135,162.192,165.33,172.249,177.091,178.559,178.758,180.585$, 180.895, 187.69, 192.661, 192.917, 195.832, 199.852, 203.297, 205.588, 209.097, 218.681, 219.639, 221.135, 224.061, 225.089, 231.432, 231.656, 241.805, 249.092, 252.972, 253.184, 269.993, 270.91, 276.443, 280.151, 281.016, 289.488, 300.299, 301.848, 304.024, 314.364, $318.997,335.848,339.955,341.136,342.52,349.235,357.381,366.838,373.402,402.126$, $408.316,423.36,423.429,432.83,445.413,459.388,461.593,472.253,504.945,521.772$, $529.951,531.566,539.326,541.759,560.236,571.51,606.559,619.012,672.537,686.757$, 705.247, 705.477, 730.141, 738.98, 812.354, 828.374, 866.997, 894.081, 897.982, 915.038, 936.333, 957.129, 996.316, 1110.47, 1135.57, 1137.9, 1224.15, 1271.92, 1331.71, 1378.13, $1524.62,1549.43,1595.51,1601.28,1718.92,1774.92,1906.84,1965.1,2092.5,2195.7$, $2334.9,2557.69,2818.65,2906.6,2954.55,3082.98,3543.66,3687.68,3832.21,4300.87$, $4315.87,4496.65,5642.23,6026.01,6570.85,6666.64,7358.75,9219.36,9499.93,10075.8$, 10533.2, 12941.1, 16897., 18035.6, 18261.3, 25000.7, 28935.4, 33698.9, 36955.4, 54518.8, $71060.4,87704.5,131517 ., 179100 ., 266419 ., 601983 ., 1.20005^{\prime} 10^{6}, 3.4545^{\prime} 10^{6}, 6.96227^{\prime} 10^{7}$ 


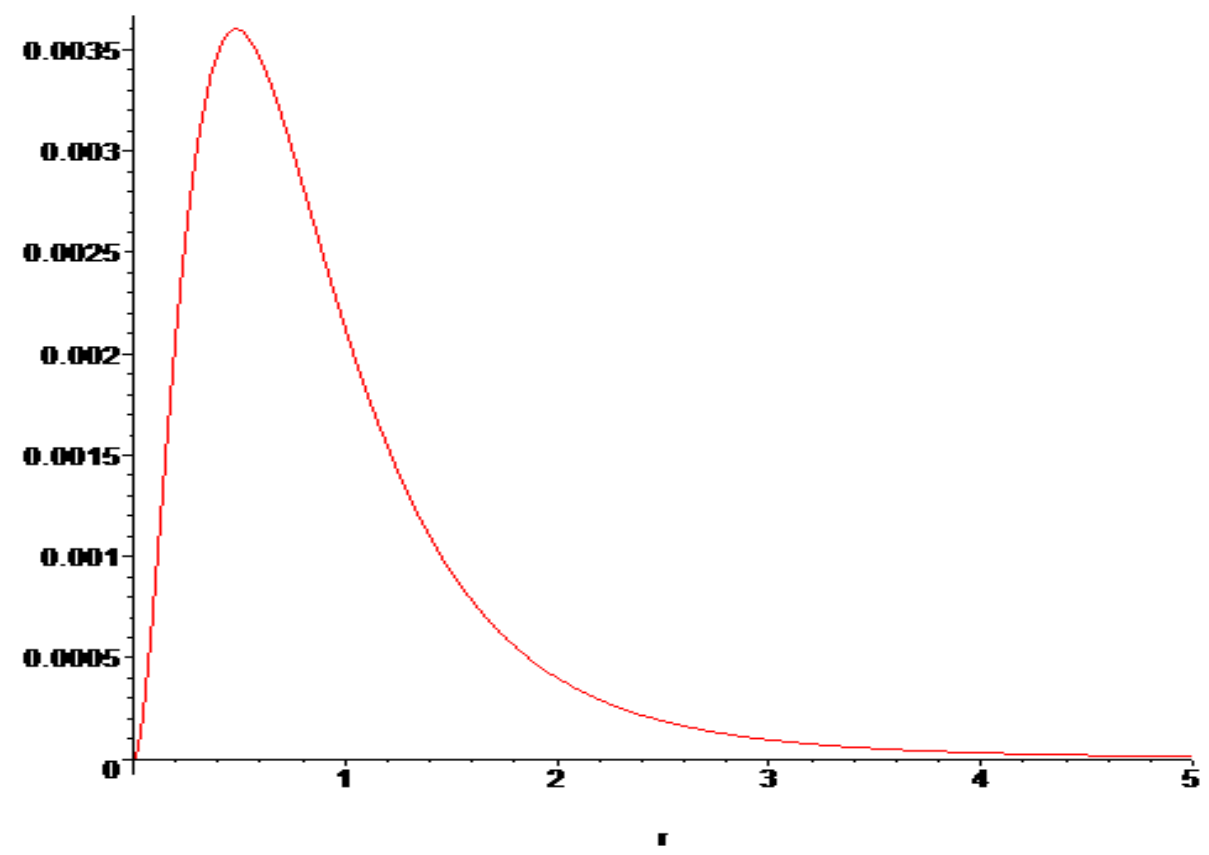

Figure 1. The plot for $U_{0,0}(r)$ 